testing. In both experiments, performance was maintained at a high level during the second test day. In the present experiment, however, performance showed a decrement 7 days after conditioning for the medium UCS intensity groups, but showed no such decrement for the high UCS intensity groups.

McAllister and McAllister (1968) have shown that there is a decrement in performance following a $47-\mathrm{h}$ interval between conditioning and test sessions. The UCS intensity used in their experiment $(.83 \mathrm{~mA})$ was comparable in magnitude to the medium UCS intensity $(.53 \mathrm{~mA})$ of the present experiment. The finding of a performance decrement 7 days after conditioning in the present experiment, with the medium UCS intensity, extends the findings of McAllister and McAllister (1968) to a longer time period after conditioning.

Further experiments are needed to explore the temporal course of the persistence of the conditioned fear response over longer time intervals such as a month after classical fear conditioning and to determine the nature of the cues responsible for the persistence of conditioned fears after conditioning with the high UCS intensity. It is quite likely that, after conditioning with the high UCS intensity, conditioned fear will persist for a longer period of time than measured in the present study. It is probable that if the test situation were made discriminably different from the conditioning situation, the conditioned fear response would extinguish rapidly.

\section{REFERENCES}

Edwards, A. L. Experimental design in psychological research. New York: Holt, Rinehart \& Winston, 1960.

Goldstein, M. L. Acquired drive strength as a joint function of shock intensity and number of acquisition trials. Journal of Experimental Psychology, 1960, 60, 349-358.

Hull, C. L. Principles of behavior. New York: A ppleton-Century-Crofts, 1943.

Lindquist, E. F. Design and analysis of experiments in psychology and education. Boston: Houghton Mifflin, 1953.

McAllister, D. E., \& McAllister, W. R. Forgetting of acquired fear. Journal of Comparative \& Physiological Psychology, $1968,65,352-355$.

McAllister, W. R., \& McAllister, D. E. Drive and reward in aversive conditioning. American Journal of Psychology, 1967, LXXX, 377-383.

Miller, N. E., \& Lawrence, D. H. Studies of fear as an acquirable drive: III. Effect of strength of electric shock as a primary drive and of number of trials with the primary drive on the strength of fear. Cited in S. S. Stevens (Ed.), Handbook of experimental psychology. New York: Wiley, 1951.

Spence, K. W. Learning and performance in eyelid conditioning as a function of the intensity of the UCS. Journal of Experimental Psychology, 1953, 45, 57-63.

Spence, K. W. Behavior theory and conditioning. New Haven, Conn: Yale University Press, 1956.

(Received for publication June 7, 1974.)

\title{
Constancy scaling theory and the Mueller-Lyer illusion: More disconfirming evidence
}

\author{
RICHARD GRIGGS*† \\ Indiana University, Bloomington, Indiana 47401
}

\begin{abstract}
Length and width judgments were made for modified Mueller-Lyer figures (the center shaft of each of the two figures was replaced by a rectangle). The rectangle with outgoing fins was judged longer and wider than a control rectangle of equal size with no fins, but longer and narrower than the rectangle with ingoing fins. The rectangle with ingoing fins was judged shorter and wider than the control rectangle. These results did not support Gregory's inappropriate constancy scaling explanation of the Mueller-Lyer illusion.
\end{abstract}

Gregory's inappropriate constancy scaling explanation has been responsible for much of the recent interest in the Mueller-Lyer illusion (Gregory, 1963, 1966, 1970). Gregory proposes that the Mueller-Lyer figures are perceived as flat projections of three-dimensional

*This research was supported by United States Public Health Service Grant PHS MH-16817 to Frank Restle. The author is grateful to Jerry Forshee for programming assistance and to Frank Restle (who sponsors the paper and takes full editorial responsibility) and Conrad Mueller for helpful comments on earlier drafts of this paper.

tThe research reported was done while the author was a graduate student at Indiana University; he is currently at the University of Florida. Requests for reprints should be sent to Richard A. Griggs, Department of Psychology, University of Florida, Gainesville, Florida 32611. displays, e.g., the figure with the outgoing fins (arrow feather figure) is a projection of an inner corner of a room and the figure with the ingoing fins (the arrowhead figure) is the projection of an outside corner of a building. Perspective cues provided by the fins in each figure cause the shaft of the arrow feather to be judged as farther away than the fins, and the shaft of the arrowhead figure to be judged closer than the fins. These cues inappropriately trigger the constancy scaling mechanism responsible for size constancy. This results in the expansion of the shaft in the arrow feather figure and the shrinkage of the shaft in the arrowhead figure producing the illusion. 
Many studies have failed to support Gregory's theory (e.g., Fisher, 1968; Pike \& Stacey, 1968; Massaro \& Anderson, 1970). (For a discussion of such evidence, see Robinson, 1972, pp. 152-162.) Some recent criticisms (Waite \& Massaro, 1970; Dengler 1972; Massaro, 1973) have centered around the use of modified Mueller-Lyer figures (the shaft of each of the component figures is replaced by a rectangle). Such figures can be seen in Fig. 1. For these figures, the inappropriate constancy scaling theory would predict that distortion should occur for judgments of both apparent width and length of the rectangles. The rectangle with outgoing fins should appear longer and wider than a rectangle with ingoing fins. This prediction was not supported by Waite and Massaro (1970) or Dengler (1972).

Using the method of reproduction, Waite and Massaro (1970) found that the rectangle bounded by outgoing fins was judged longer and narrower than the rectangle bounded by ingoing fins. However, all mean length and width judgments were underestimations of the actual lengths and widths. Control rectangles without any inducing fins were not used to provide a baseline so it is not clear what these underestimations and results mean.

Dengler (1972), using the method of adjustment, did employ reference rectangles without fins. The rectangle with outgoing fins was judged longer and narrower than a reference rectangle while the rectangle with ingoing fins was judged shorter and wider than a reference rectangle. Unlike Waite and Massaro (1970), length and width judgments were not all underestimations of real length and width.

If the stimulus dimensions that are optimal for typical Mueller-Lyer figures are also best for the modified figures, there is some question about whether the dimensions employed by Dengler were optimal. Dengler employed a fin length of $4.5 \mathrm{~cm}$ and rectangular lengths of 7.0 and $8.0 \mathrm{~cm}$. Thus, the fin length was approximately $56 \%$ and $64 \%$ of the rectangular lengths.

The magnitude of the Mueller-Lyer illusion is directly related to fin length (Heymans, 1896; Lewis, 1909;
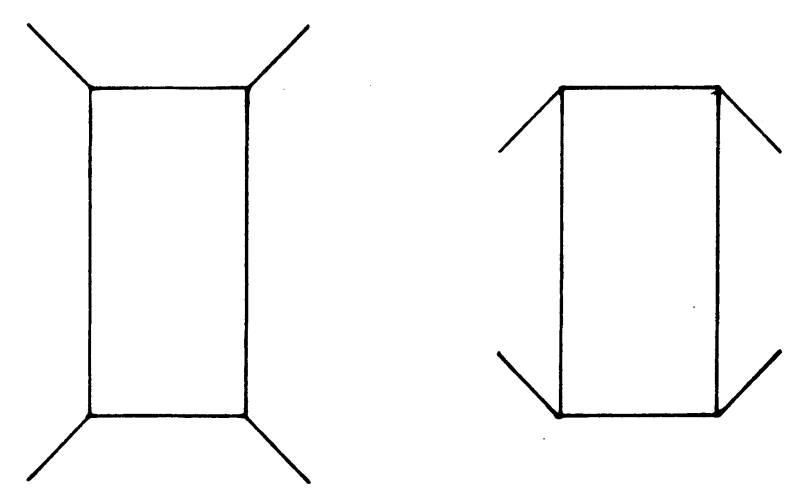

Fig. 1. Examples of modified Mueller-Lyer figures in which the shaft of each component is replaced by a rectangle.
Dewar, 1967). Lewis (1909) and Heymans (1896) found that, as fin length increased (with length of shaft and angle between the fins held constant), the illusion increased to a maximum and then decreased. Heymans (1896) found a maximal illusion when the fins were about $25 \%$ the length of the shaft and the angle between the fins as small as possible. Nakagawa (1958) reported maximum distortion when fin length was about $33 \%$ of the length of the shaft. However, estimates of this maximum are not all in agreement. Different versions of the Mueller-Lyer illusion have produced maximum illusions for different fin lengths. Hayami and Miya (reported by Oyama, 1960) report a maximum for the figure with outgoing fins at a fin length that is $50 \%$ the length of the shaft, and one of $33 \%$ for the figure with ingoing fins. However, they studied the version of the Mueller-Lyer with shafts deleted. This finding for the arrow feather case is close to the fin length/shaft ratios used by Dengler but with very different figures.

It could be argued, as Krueger (1972) does, that "The depth cue produced by the outgoing fins ought to become ever larger as the fins are lengthened, rather than to increase at first but then to become weaker as fin length passes a particular value." If this were so, the stimulus dimensions employed by Dengler would seem very appropriate. However, as Gregory (1972) points out, Kreuger's expectation has not been shown and may only be due to a misunderstanding of "depth-cue scaling." (For more detail, see Gregory, 1972).

The present study investigated the Mueller-Lyer illusion using the modified rectangular figures discussed above but with what would seem to be more optimal stimulus dimensions, control rectangles, and a different method of illusion measurement. It was hoped that such changes would provide a fairer test of Gregory's inappropriate constancy scaling theory.

\section{METHOD}

\section{Subjects}

Forty undergraduate students in introductory psychology at Indiana University served as Ss in partial fulfillment of course requirements. Ss were run in groups of up to four at a time. Each such group was assigned to one of two conditions, resulting in 20 Ss in each condition.

\section{Apparatus and Materials}

Stimuli consisted of 27 slides, each showing a white outline of a rectangle with outgoing fins, ingoing fins, or no fins. Each figure was centered and the background of the slide was black. Fins and the sides of the rectangles were approximately $1 \mathrm{~cm}$ in thickness. Three lengths $(23.5,26.5$, and $29.5 \mathrm{~cm})$ and three widths $(7.5,10.5$, and $13.5 \mathrm{~cm})$ were combined factorially with the three types of figures to form the 27 slides. The fins were $5 \mathrm{~cm}$ in length and were tilted, outward or inward, $45 \mathrm{deg}$ from the vertical.

The slides were projected from a Kodak Carousel RA-950 random access slide projector onto a screen approximately $3 \mathrm{~m}$ from Ss. The screen was $130 \times 130 \mathrm{~cm}$ and its edges were masked so as to contrast little with the wall behind. 


\section{Procedure}

The method of single stimuli was employed. On each trial S was shown a slide and he made a judgment of the length (or width) of the central vertical axis of the rectangle (or horizontal axis in the case of a width judgment) by pressing one of a row of six buttons. The leftmost button corresponded to "very long," the rightmost button to "very short," and the other four buttons to "intermediate lengths."

Two groups were used. Ss in Group 1 made length judgments for five repetitions, received a short break during which the width judgment instructions were given, and then made width judgments for five repetitions. The order of types of judgments was reversed for Ss in Group 2 to check for a posible order effect. Slides were shown one at a time in random order and remained in view until all Ss had responded or for $5 \mathrm{sec}$. A 3-sec intertrial interval was used. Ss viewed all 27 slides five times in five different random permutations for each type of judgment (length and width).

All programming of stimuli, presentation of slides, and data collection were done by an IBM 1800 process-control computer.

\section{RESULTS}

The six buttons from left to right were assigned the numbers $1-6$, respectively. The first repetition for each type of judgment in both conditions was treated as practice and only the last four length and width judgments to each slide were used in the analyses. Two repeated-measures analyses of variance run on the combined length data of Groups 1 and 2 and on the combined width data of Groups 1 and 2 revealed no significant differences in order of judgment type (length-width vs width-length). Hence, subsequent analyses were done on the combined data for each type of judgment.

Mean judgments of length and width as a function of type of figure are given in Table 1. Statistical tests were performed using two-tailed Dunnett tests. The mean length judgment for a rectangle with outgoing fins is significantly greater $(p<.01)$ and the mean length judgment for a rectangle with ingoing fins is significantly less $(p<.05)$ than the mean length judgment for a control rectangle. The mean width judgments for a rectangle with outgoing fins and for a rectangle with ingoing fins are both significantly greater than the mean width judgment for a control rectangle, $(p<.05)$ and $(\mathrm{p}<.01)$, respectively. However, the mean width judgment for a rectangle with outgoing fins is significantly less $(p<.01)$ than the mean width judgment for a rectangle with ingoing fins. Thus, rectangles with outgoing fins are judged longer but narrower than rectangles with ingoing fins.

\section{DISCUSSION}

As Massaro (1973) points out, Gregory's inappropriate constancy scaling explanation of the Mueller-Lyer illusion would almost be untestable unless we require that the depth cues operate consistently within a given illusory situation. Thus, the distortion of the perceived length and width of the modified figures should be in the same direction (the rectangle with outgoing fins should be judged longer and wider than the rectangle with ingoing fins). The present results do not support this prediction. The rectangle with outgoing fins is judged longer
Table 1

Mean Judgments of Length and Width as a Function of Type of Figure

\begin{tabular}{lll}
\hline & \multicolumn{2}{c}{ Mean Judgment } \\
\cline { 2 - 3 } Type of Figure & Length & Width \\
\hline Rectangle With Outgoing Fins & $4.637^{* *}$ & $3.189^{*}$ \\
Rectangle With Ingoing Fins & $3.935^{*}$ & $3.506^{* *}$ \\
Control Rectangle With No Fins & 4.045 & 3.075 \\
\hline
\end{tabular}

Note-Asterisks indicate mean judgment is significantly different from mean judgment for control rectangle.

${ }^{*} p<.05 \quad * * p<.01$

but narrower than the rectangle with ingoing fins. This result agrees with the findings of both Waite and Massaro (1970) and Dengler (1972). The inability of this theory to account for the width judgments in all three studies casts great doubt upon its validity.

Gregory (1973), however, argues that such studies do not show that depth-cue scaling does not function transversely in the Mueller-Lyer (depth-cue scaling is operating for the space between the fins but not transverse to a line between them), and even if this difference did exist between depth-cue scaling and depth-hypothesis scaling (depth-hypothesis scaling does operate transversely), it would not imply a logical inconsistency in the inappropriate constancy scaling theory. Thus, it seems Gregory is arguing that the depth cues do not have to operate consistently within a given illusory situation. If not, the inappropriate constancy scaling theory would appear to be essentially untestable as Humphrey and Morgan (1965) have suggested.

\section{REFERENCES}

Dengler, M. A test of constancy scaling theory in a modified Mueller-Lyer illusion. Perception \& Psychophysics, 1972, 12 339-341.

Dewar, R. E. Stimulus determinants of the magnitude of the Müller-Lyer illusion. Perceptual \& Motor Skills, 1967, 24, 708-710.

Fisher, G. H. An experimental and theoretical appraisal of the inappropriate size-depth theories of illusion. British Journal of Psychology, 1968, 59, 373-383.

Gregory, R. L. Distortion of visual space as inappropriate constancy scaling. Nature, 1963, 199, 678-680.

Gregory, R. L. Eye and brain. New York: World University Press, 1966.

Gregory, R. L. The intelligent eye. London: Weidenfeld, 1970.

Gregory, R. L. Comments on L. E. Krueger's "Disconfirming evidence"' of R. L. Gregory's theory of illusions. Psychological Review, 1972, 79, 540-541.

Gregory, R. L. Reply to Massaro. Psychological Review, 1973, 80,304 .

Heymans, G. Quantitative untersuchunger über das "optische Paradoxen," Zeitschrift für Psychologie, 1896, 9, 221-255.

Humphrey, N. K., \& Morgan, M. J. Constancy and the geometric illusions. Nature, $1965,206,744-745$.

Krueger, L. E. Gregory's theory of illusions: Some disconfirming evidence in the case of the Müller-Lyer illusion. Psychological Review, 1972, 79, 538-539.

Lewis, E. O. Confluxion and contrast in the Müller-Lyer illusion. British Journal of Psychology, 1909, 3, 21-41.

Massaro, D. W. Constancy scaling revisited. Psychological R eview, 1973, 80, 303.

Massaro, D. W. \& Anderson, N. H. A test of a perspective theory of geometrical illusions. The American Journal of Psychology, $1970,83,567-575$.

Nakagawa, D. Müller-Lyer illusion and retinal induction. Psychologia, 1958, 1, 167-174.

Oyama, T. Japanese studies on the so-called geometrical optical illusions. Psychologia, 1960, 3, 7-20.

Pike, A. R. and Stacey, B. G. The perception of luminous Müller-Lyer Figures and its implications for misapplied constancy theory. Life Sciences, 1968, 7, 355-362.

Robinson, J. O. The psychology of visual illusion. London: Hutchinson \& Co., 1972.

Waite, H., \& Massaro, D. W. Test of Gregory's constancy scaling explanation of the Müller-Lyer illusion. Nature, 1970, 227 733-734.

(R eceived for publication July 13, 1974.) 